\title{
Performance evaluation of Modified Widrow-Hoff ADALINE under effect of photovoltaic with shunt active power filter
}

\begin{abstract}
This paper investigates performances of Modified Widrow-Hoff ADALINE as harmonics extraction algorithm under effect of photovoltaic (PV) integrated with shunt active power filter (SAPF). Integration of PV with SAPF gives high advantages in terms of energy source and power quality. The dependable on grid supply current reduces as PV current may support good amount of current to the load. However, with off-on operation and various irradiance levels of the PV, a good harmonics extraction algorithm is needed. Meanwhile, Modified Widrow-Hoff ADALINE is already proven before in SAPF functionality, however, its performance should further be evaluated under integration of PV with SAPF. Therefore, this algorithm is proposed, and further designed and developed for PV based SAPF, as simulated in MATLAB/Simulink. From the simulation results, the Modified Widrow-Hoff algorithm shows good performances for PV based SAPF with low THD values, fast response times and reduced source power from the grid under various levels of irradiance.
\end{abstract}

Keyword: Active power filter; Artificial neural network; Harmonics; Photovoltaic; Power quality 